\title{
Intrathecal morphine versus epidural ropivacaine infusion for analgesia after Cesarean section: a retrospective study
}

Hiroko Suzuki ${ }^{1,2}$, Yoshinori Kamiya ${ }^{1,3^{*}}$, Takashi Fujiwara ${ }^{2}$, Takayuki Yoshida ${ }^{1}$, Misako Takamatsu ${ }^{1}$ and Kazunori Sato ${ }^{2}$

\begin{abstract}
Background: Analgesia after Cesarean delivery (CD) requires early ambulation to prevent thromboembolic disease and to facilitate baby care. We retrospectively reviewed anesthesia charts and medical records of patients who underwent CD to compare the efficacy of spinal anesthesia supplemented with intrathecal morphine hydrochloride (ITM) and combined spinal-epidural anesthesia followed by opioid-free epidural analgesia (CSEA-EDA).

Findings: All subjects underwent CD at Nagaoka Chuo General Hospital between February 2012 and January 2013. Patient characteristics, time to first analgesic rescue after $C D$, and analgesic use after $C D$ were examined. Incidences of postural hypotension, lower extremity numbness/weakness, postoperative nausea/vomiting (PONV), and pruritus were also examined for $48 \mathrm{~h}$ after CD. Average time to first analgesic use after CD (ITM 25.13 $\pm 16.07 \mathrm{~h}$, CSEA-EDA $22.42 \pm 16.27 \mathrm{~h}, p=0.521$ ) and cumulative probability of rescue analgesic use ( $p=0.139$ by log-rank test) were comparable between groups. However, average analgesic use within $24 \mathrm{~h}$ was lower in the ITM group $0.75 \pm 1.05$ times) than in the CSEA-EDA group (1.52 \pm 1.72 times, $p=0.0497)$. Numbness or motor weakness in lower extremities only occurred in the CSEA-EDA group, and pruritus only occurred in the ITM group.
\end{abstract}

Conclusions: The results of this study suggest that ITM is better than CSEA-EDA for anesthesia following CD with regard to pain control. Also, ITM would be advantageous for early ambulation following CD because of lower incidence of numbness and motor weakness in lower extremities compared to CSEA-EDA.

Keywords: Cesarean delivery; Spinal anesthesia with intrathecal morphine; Opioid-free epidural analgesia; Postoperative pain; Adverse effects

\section{Findings Introduction}

A Cesarean section is one of the most commonly performed surgical procedures. Combined spinal-epidural anesthesia (CSEA) is a favorable neuraxial anesthetic technique for Cesarean deliveries (CD) because the epidural catheter can also be utilized for postoperative patientcontrolled epidural analgesia. However, postoperative epidural analgesia frequently causes lower extremity numbness and weakness, which can delay early ambulation $[1,2]$.

\footnotetext{
* Correspondence: y-kamiya@med.niigata-u.ac.jp

1 Division of Anesthesiology, Niigata University Graduate School of Medical and Dental Sciences, 1-757 Asahimachi-dori, Chuo Ward, Niigata, Niigata 951-8510, Japan

${ }^{3}$ Present address: Department of Anesthesiology, Uonuma Institute of Community Medicine, Niigata University Medical and Dental Hospital, 4132 Urasa, Minami-uonuma, Niigata 949-7302, Japan

Full list of author information is available at the end of the article
}

Opioid-supplemented epidural analgesia after CD can provide superior analgesia and has a low incidence of lower extremity numbness and weakness [3, 4]. However, opioid supplementation in continuous epidural analgesia can lead to postoperative nausea and vomiting (PONV), which hinders early ambulation and recovery. Moreover, in the perinatal period, women are more susceptible to thromboembolic disease, and perioperative anticoagulant therapy is recommended to prevent thromboembolic disease during pregnancy. Unfortunately, anticoagulants can make epidural catheter handling more difficult.

Morphine-supplemented spinal anesthesia [5], or single-shot intrathecal morphine (ITM), can rapidly induce anesthesia and provide long-term postoperative pain relief for elective and emergency CDs. Therefore, ITM may allow for early ambulation after $C D$, which reduces the risk thromboembolic disease and facilitates 
baby care. Unfortunately, intrathecal opioids are associated with PONV, pruritus, sedation, and respiratory depression [5, 6].

Numerous clinical studies have evaluated the efficacy of ITM and CSEA followed by epidural analgesia in patients undergoing $\mathrm{CD}$. To the best of our knowledge, no study has directly compared anesthesia efficacy and adverse effect incidences between ITM and CSEA with opioid-free epidural analgesia (CSEA-EDA) within $48 \mathrm{~h}$ of $\mathrm{CD}$. We retrospectively examined and compared the efficacy and safety of spinal anesthesia with ITM and CSEA-EDA after CD.

\section{Methods}

\section{Study subjects}

This retrospective cohort study was performed at Nagaoka Chuo General Hospital. The study was reviewed and approved by the Nagaoka Chuo General Hospital institutional review board. Anesthesia charts and medical records of consecutive patients who underwent $\mathrm{CD}$ between February 2012 and January 2013 were reviewed. Patients that underwent spinal anesthesia without ITM or general anesthesia for $\mathrm{CD}$ were excluded from analyses.

\section{Analgesic methods}

The ITM group received $0.5 \%$ hyperbaric bupivacaine (8.5-12 mg) supplemented with $100 \mu \mathrm{g}$ of intrathecal preservative-free morphine hydrochloride. The CSEAEDA group had an epidural catheter inserted at the T11/ 12, T12/L1, or L1/2 intervertebral space using standard procedures. After test dose administration $(3 \mathrm{~mL}$ bolus of $1 \%$ mepivacaine), $0.5 \%$ hyperbaric bupivacaine (3.5$9.0 \mathrm{mg}$ ) was administered into the intrathecal space. Epidural $0.75 \%$ ropivacaine $(5-12 \mathrm{~mL})$ was given as needed to obtain a block of adequate height. Immediately after surgery, $0.2 \%$ ropivacaine was administered through the epidural catheter $(4 \mathrm{~mL} / \mathrm{h})$ using a disposable infuser (Coopdeck Baloonjector 300 with PCA apparatus, Daiken Medical, Osaka, Japan). The CSEA-EDA group had anesthesia administered by one physician (KS), and the ITM group had anesthesia administered by two physicians (HS or TF).

All patients were mobilized 6-8 h after surgery. Based on pain level and patient requests, rescue analgesics were administered to manage postoperative breakthrough pain (numeric rating scale $[N R S] \geq 3-4$ ). Patients were first administered suppository diclofenac sodium $(50 \mathrm{mg}$ ) or oral loxoprofen sodium $(60 \mathrm{mg})$. The CSEA-EDA group had the option of patient-controlled epidural anesthesia (PCEA; $0.2 \%$ ropivacaine $3 \mathrm{~mL}$, lockout interval of $60 \mathrm{~min}$ ) for initial rescue analgesic administration. If pain persisted, intramuscular pentazocine $(30 \mathrm{mg})$ was given. Acetaminophen $(400 \mathrm{mg}$ ) was used as an alternative or supplementation to NSAID analgesia, if needed.
Data were collected within $48 \mathrm{~h}$ of CD and included patient characteristics, time to first rescue analgesic use, rescue analgesic use frequency, and adverse event (postural hypotension, lower extremity numbness and weakness, PONV, and pruritus) incidences. Primary outcomes included time to first rescue analgesic use and rescue analgesic use frequency. Secondary outcomes were the incidences of adverse effects within $48 \mathrm{~h}$ of CD.

\section{Data analyses}

Continuous variables are presented as mean \pm standard deviation. Categorical variables, ordinal variables, and non-normally distributed data are presented as median (range). All statistical analyses were performed using Microsoft Excel 2011 for Macintosh (Microsoft, Redmond, WA, USA) with a statistical macro (XLSTAT 2014; Addinsoft, New York, NY, USA). An unpaired $t$ test or Mann-Whitney $U$-test was used to determine statistical significance of differences in patient characteristics. A logrank test was used to compare cumulative probabilities. A Kolmogorov-Smirnov test was used to compare time distribution of rescue analgesic use. Patients who did not require rescue analgesics were assigned a first use time of $48 \mathrm{~h}$. The comparisons of frequency of rescue analgesic use within $48 \mathrm{~h}$ after $\mathrm{CD}$ and the average time to first rescue analgesic use between groups in which the epidural puncture was performed were made using Kruskal-Wallis test and one-way analysis of variance (ANOVA), respectively. The comparisons between groups in which epidural analgesia was continued or discontinued within $48 \mathrm{~h}$ after $\mathrm{CD}$ were performed using Mann-Whitney $U$-test and unpaired $t$ test, respectively. Incidences of anesthesia-related adverse effects were compared using Fisher's exact tests. Statistical significance was defined as $p<0.05$.

\section{Results}

Eighty-five patients underwent CDs which occurred during the study period, of which 32 and 27 patients were included in the ITM and CSEA-EDA groups, respectively. Patient demographic characteristics are summarized in Table 1. No significant differences between groups were observed in age, height, body weight, gestational week, and emergency $\mathrm{CD}$ rate. The number of cases per site of epidural puncture in CSEA procedure was as follows: T11/12, $n=8$; T12/L1, $n=12$; and $\mathrm{L} 1 / 2, n=7$. Average time to first rescue analgesic use (including patient-controlled local anesthetic bolus infusion in the CSEA-EDA group) was not statistically different between groups (ITM $25.13 \pm 16.07 \mathrm{~h}$, CSEA-EDA $22.42 \pm 16.27 \mathrm{~h}, p=0.521$, unpaired $t$ test). Moreover, the cumulative probability of rescue analgesic and/or PCEA use was not significantly different between groups (Fig. 1). The percentage of patients using rescue analgesics within $48 \mathrm{~h}$ of $\mathrm{CD}$ was similar between groups (ITM 25 of 32 patients [78.1\%], CSEA-EDA 22 of 27 
Table 1 Demographic data of the patients included in this study

\begin{tabular}{llll}
\hline & $\begin{array}{l}\text { ITM group } \\
(n=32)\end{array}$ & $\begin{array}{l}\text { CSEA-EDA group } \\
(n=27)\end{array}$ & $p$-value \\
\hline Age (years) & $33.6 \pm 5.2$ & $31.4 \pm 4.1$ & 0.24 \\
Height $(\mathrm{cm})$ & $158 \pm 5.3$ & $157 \pm 4.1$ & 0.30 \\
Weight $(\mathrm{kg})$ & $64.3 \pm 9.3$ & $63.4 \pm 8.9$ & 0.62 \\
Gestational week & $38.0 \pm 1.7$ & $38.5 \pm 2.1$ & 0.55 \\
Emergency CD incidence & $11(34 \%)$ & $12(44 \%)$ & 0.30 \\
\hline
\end{tabular}

Data are shown as mean \pm SD or as actual incidence number (percentage of total patients). An unpaired $t$ test was used to analyze the age, height, weight, and gestational week; Fisher's exact test was used to analyze the incidences of emergency $C D$

ITM intrathecal morphine-supplemented spinal anesthesia, CSEA-EDA combined spinal-epidural anesthesia followed by opioid-free epidural analgesia, $C D$ Cesarean delivery

patients [81.5\%]). The frequency of rescue analgesic use was significantly lower in the ITM group (0 time [range 0$3]$ ) than in the CSEA-EDA group (1 time [0-6], $p=0.0497$ ) within $24 \mathrm{~h}$ of CD, but not in the 24-48 h after CD (ITM 2 times [0-4], CSEA-EDA 1 time [0-5], $p=0.465$; Fig. 2). The frequency of rescue analgesic use within $48 \mathrm{~h}$ of CD and the average time to first rescue analgesic use were not significantly different between the groups in which the epidural puncture was performed (Table 2).

Analgesia-related adverse effects occurred within $48 \mathrm{~h}$ in both groups. Lower extremity numbness and weakness

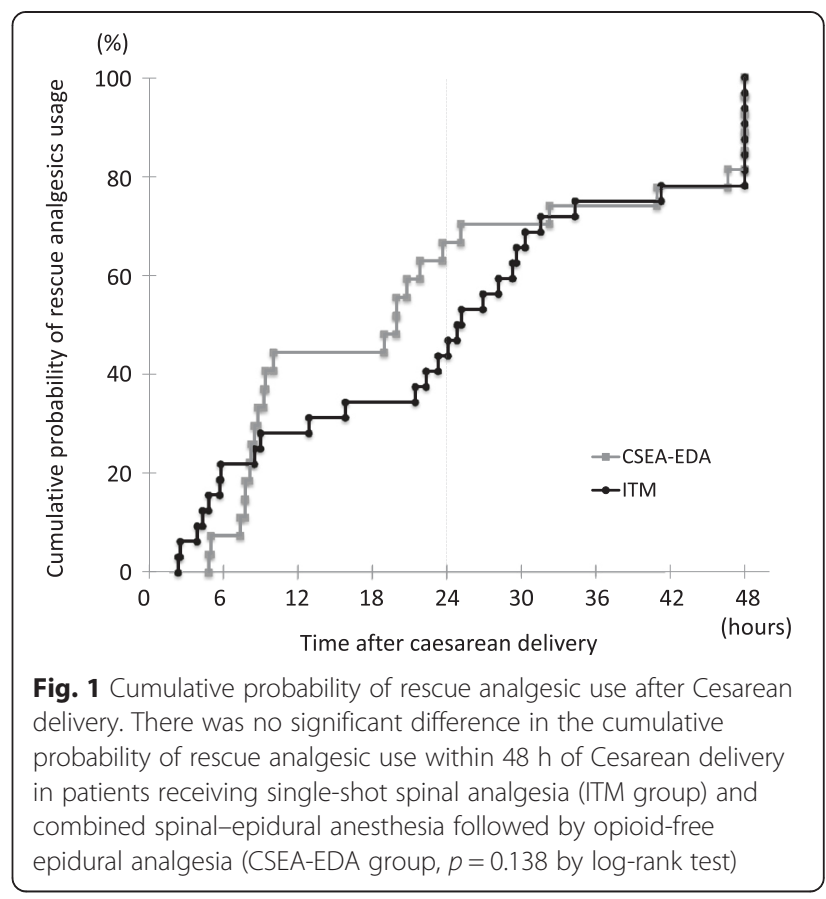

only occurred in the CSEA-EDA group, and pruritus only occurred in the ITM group (Table 3 ). The incidence of numbness or motor weakness in CSEA-EDA group was as follows: T11/12, $n=2$; T12/L1, $n=4$; and L1/2, $n=3$. The odds ratio of incidence of numbness or motor weakness
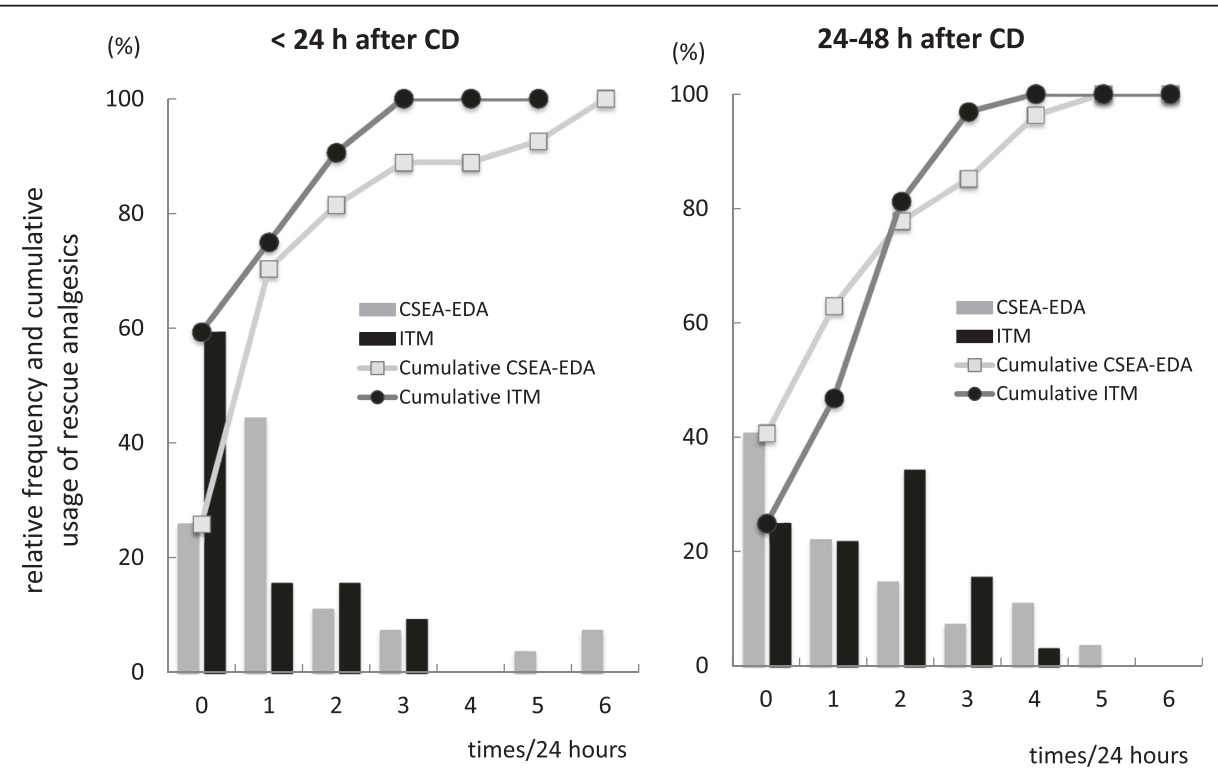

Fig. 2 Relative frequency of rescue analgesic usage within $24 \mathrm{~h}$ of Cesarean delivery (CD) and between 24 and $48 \mathrm{~h}$ after CD in patients receiving single-shot spinal analgesia (ITM group) and combined spinal-epidural anesthesia followed by opioid-free epidural analgesia (CSEA-EDA group). Within $24 \mathrm{~h}$ of CD, the relative frequency and distribution of rescue analgesic use in the ITM group (ITM 0 times [range 0-3]) was lower than that in the CSEA-EDA group ( 1 time [0-6], $p=0.0497$ by Mann-Whitney U-test, $p=0.019$ by Kolmogorov-Smirnov test). Rescue analgesic use between 24 and $48 \mathrm{~h}$ after CD was not significantly different between groups (ITM 2 times [0-4], CSEA-EDA 1 time [0-5], $p=0.416$ by Mann-Whitney $U$-test, $p=0.465$ by Kolmogorov-Smirnov test). Vertical bar charts represent the relative frequency of rescue analgesic use in each group. Line plots represent the cumulative frequency of rescue analgesic use in each group. Data are presented as median [range] 
Table 2 Frequency of rescue analgesic use and average time to first rescue analgesic use within $48 \mathrm{~h}$ after CD between the groups, in which the epidural puncture was performed

\begin{tabular}{llllll}
\hline & & T11/12 & T12/L1 & L1/2 & p-value \\
& & $(8 / 27 ;$ & $(12 / 27 ;$ & $(7 / 27 ;$ & \\
& $29.6 \%)$ & $44.4 \%)$ & $25.9 \%)$ & \\
\hline $\begin{array}{l}\text { Frequency of rescue } \\
\text { analgesic use after CD } \\
\text { (times) }\end{array}$ & $<24 \mathrm{~h}$ & $1[0-6]$ & $0[0-3]$ & $1[0-5]$ & 0.59 \\
& $24-$ & $0[0-3]$ & $1[0-4]$ & $2[0-5]$ & 0.09 \\
& $48 \mathrm{~h}$ & & & & \\
& $<48 \mathrm{~h}$ & $1[0-9]$ & $2[0-6]$ & $4[1-$ & 0.1 \\
& & & & $11]$ & \\
$\begin{array}{l}\text { Average time to first rescue } \\
\text { analgesic use (hh:mm) }\end{array}$ & $28: 47 \pm$ & $19: 12 \pm$ & $20: 38 \pm$ & 0.43 \\
\hline
\end{tabular}

Data are shown as median [range] or mean+/-SD. Statistical analyses were performed by Kruskal-Wallis test for frequency of rescue analgesic use and by oneway ANOVA for average time to first rescue analgesic use within $48 \mathrm{~h}$ after CD $C D$ Cesarean delivery

was not significantly different between the groups in which the epidural puncture was performed. Postural hypotension occurred more often in the CSEA-EDA group, but the difference was not statistically significant $(p=0.08)$. Five of 27 patients $(18.5 \%)$ in the CSEA-EDA group had epidural catheter issues (i.e., connector trouble or local anesthetic leakage from catheter insertion site). Catheter issues led to obstetrician removal of the catheter within $48 \mathrm{~h}$ of $\mathrm{CD}$ in 12 of 27 patients (44.4\%) in the CSEA-EDA group. The frequency of rescue analgesic use within $24 \mathrm{~h}$ of $\mathrm{CD}$ and the average time to first rescue analgesic use were not significantly different between groups that discontinued epidural analgesia within $48 \mathrm{~h}$ of CD and those that continued analgesia (Table 4). Respiratory depression was not observed in any patient in either group.

\section{Discussion}

This small retrospective study revealed that the ITM group required fewer analgesic interventions than the CSEA-EDA group within $24 \mathrm{~h}$ of CD. Additionally, fewer adverse effects that impeded early ambulation (lower extremity numbness and weakness, orthostatic hypotension) occurred in the ITM group than in the CSEA-EDA group.
Table 3 Incidence of adverse effects associated with each anesthetic technique

\begin{tabular}{llll}
\hline & $\begin{array}{l}\text { ITM group } \\
(n=32)\end{array}$ & $\begin{array}{l}\text { CSEA-EDA } \\
\text { group }(n=27)\end{array}$ & $\begin{array}{l}p \text { - } \\
\text { value }\end{array}$ \\
\hline Postural hypotension & $2(6.3 \%)$ & $6(22.2 \%)$ & 0.08 \\
PDPH & - & $2(7.4 \%)$ & 0.21 \\
Numbness in the lower & - & $9(33.3 \%)$ & $<0.001$ \\
extremities 24 h after CD & & & \\
PONV & $2(6.3 \%)$ & $2(7.4 \%)$ & 0.63 \\
Pruritus & $12(37.5 \%)$ & - & $<0.001$ \\
Catheter trouble & - & $5(18.5 \%)$ & - \\
\hline
\end{tabular}

Data are shown as actual incidence number (percentage of total patients). Statistical analyses were performed by Fisher's exact test $C D$ Cesarean delivery, ITM intrathecal morphine-supplemented spinal anesthesia, CSEA-EDA combined spinal-epidural anesthesia followed by opioid-free epidural analgesia, $P D P H$ postdural puncture headache, $P O N V$ postoperative nausea and vomiting

Intrathecal opioids are effective for managing postoperative pain after $\mathrm{CD}$ but are associated with pruritus, PONV, and respiratory depression [5-7]. Lower extremity numbness more than $24 \mathrm{~h}$ after $\mathrm{CD}$ and respiratory depression were not observed in the ITM group. Additionally, PONV incidence in the ITM group was not statistically different than in the CSEA-EDA group. Previous studies have shown that respiratory depression is very rare in patients undergoing $C D$ with morphine-supplemented spinal anesthesia [8]. Moreover, $0.1 \mathrm{mg}$ of morphine sufficiently reduces postoperative pain with little respiratory depression $[5,9]$.

Previous studies demonstrated that CSEA-EDA adequately manages postoperative pain after $\mathrm{CD}$ and has a low incidence of adverse effects (PONV and hypotension) $[10,11]$. However, early ambulation after CD is important to prevent thromboembolic diseases and to facilitate infant breastfeeding and care. Therefore, postoperative epidural analgesia may be disadvantageous because epidural analgesia with local anesthetics causes lower extremity numbness and weakness. Previous reports found lower extremity weakness incidences of $26 \%$ [3] and 11-41\% [11] after $\mathrm{CD}$ and $11.5 \%$ after gynecological and urological surgery [12] when postoperative epidural analgesia was used. Furthermore, lower extremity numbness incidences

Table 4 Frequency of rescue analgesic use and average time to first rescue analgesic use within $48 \mathrm{~h}$ after CD between the groups, in which the postoperative epidural analgesia was continued for $48 \mathrm{~h}$ or was discontinued

\begin{tabular}{|c|c|c|c|c|}
\hline & & $\begin{array}{l}\text { Continued epidural analgesia } \\
(15 / 27 ; 55.6 \%)\end{array}$ & $\begin{array}{l}\text { Discontinued epidural analgesia } \\
(12 / 27 ; 44.4 \%)\end{array}$ & $p$-value \\
\hline \multirow[t]{3}{*}{ Frequency of rescue analgesic use after CD (times) } & $<24 \mathrm{~h}$ & $1[0-6]$ & $1[0-6]$ & 0.5 \\
\hline & $24-48 \mathrm{~h}$ & $0.5[0-4]$ & $2[0-5]$ & 0.2 \\
\hline & $<48 \mathrm{~h}$ & $1.5[0-9]$ & $2[0-11]$ & 0.61 \\
\hline Average time to first rescue analgesic use (hh:mm) & & $21: 16 \pm 14: 33$ & $23: 28 \pm 18: 12$ & 0.73 \\
\hline
\end{tabular}

Data are shown as median [range] or mean+/-SD. Statistical analyses were performed by Mann-Whitney $U$-test for frequency of rescue analgesic use and by unpaired $t$ test in average time to first rescue analgesic use within $48 \mathrm{~h}$ after $C D$ $C D$ Cesarean delivery 
were $42 \%$ after gynecological surgery [13], 37.5-67.5 \% after CD [4], and $35.5 \%$ after gynecological and urological surgeries [12] when postoperative epidural analgesia was used. In our study, numbness or motor weakness of lower extremities occurred in $33.3 \%$ of patients in the CSEAEDA group, and the site of epidural catheter insertion was not correlated with this adverse effect. This result is comparable to that of the previous studies listed above. In most cases, CSEA was performed with CSEA needle and puncture site was L2/3-L4/5. In such cases, incidence of numbness or motor weakness of lower extremities would be expected to be much higher than that observed in our study.

In this study, none of the patients in the CSEA-EDA group were converted to general anesthesia or to intraoperative supplemental administration of local anesthetics. Therefore, we concluded that the patients in both groups received adequate anesthesia for $\mathrm{CD}$. In addition, although the average dose of spinal bupivacaine in the CSEA-EDA group was significantly lower than that in the ITM group, as shown in Anesthetic Methods (ITM group $9.75 \pm$ $0.87 \mathrm{mg}$, CSEA-EDA $5.42 \pm 0.97 \mathrm{mg}, p<0.001$, MannWhitney $U$-test), the average time to first rescue analgesic use in the CSEA-EDA group was comparable to that in the ITM group. These results suggested that epidural anesthesia was effective for managing postoperative pain after CD. There was no statistical significance in analgesic efficacies between different intervertebral spaces of epidural puncture site.

Moreover, we also found that $44 \%$ of CSEA-EDA patients had discontinued epidural analgesia within $48 \mathrm{~h}$ of $\mathrm{CD}$ because of lower extremity numbness and weakness $(33.3 \%)$ and/or catheter trouble (18.5\%). When epidural analgesia is discontinued, another rescue analgesic is needed to manage postoperative pain. However, analgesic efficacies within $24 \mathrm{~h}$ of CD were not significantly different between the groups that discontinued epidural analgesia within $48 \mathrm{~h}$ of $\mathrm{CD}$ and those that continued analgesia. Postoperative analgesia in the early period after CD might be crucial for postoperative pain control.

Though pruritus is not life-threatening, it can severely impact recovery quality in $\mathrm{CD}$ patients administered with opioids. Pruritus only occurred in the ITM group with a similar incidence as previously reported [14-16]. Unfortunately, our retrospective data do not allow us to comment on how pruritus affected postpartum recovery.

\section{Conclusions}

This retrospective cohort study had several limitations. First, patient pain was not evaluated using a subjective scale because this information was not in medical records. Therefore, postoperative pain was evaluated with supplemental analgesic use. Second, retrospective studies carry inherent biases because they are not randomized or blinded. Lastly, our study included a relatively small number of subjects.

In conclusion, our study suggests that ITM is as effective as CSEA-EDA in managing postoperative pain after CD, but ITM does not inhibit early ambulation. Unfortunately, pruritus can occur with ITM, and countermeasures to prevent this should be considered.

\section{Abbreviations}

CD: Cesarean delivery; CSEA: combined spinal-epidural anesthesia; EDA: epidural analgesia; ITM: intrathecal morphine; PONV: postoperative nausea and vomiting.

\section{Competing interests}

The authors declare that they have no competing interests.

\section{Authors' contributions}

HS and TF conceived of and designed the study. HS, TF, and KS collected the data. HS and YK interpreted the data and wrote the manuscript. TY and MT provided valuable discussion of the results and critically reviewed the manuscript. All authors have read and approved the final manuscript.

\section{Acknowledgements}

Financial support was solely provided by the institution and/or department. We wish to thank Dr. Tatsunori Watanabe for the study appraisal and for providing constructive advice on this manuscript. We also thank Editage ${ }^{\bullet}$ for providing editorial assistance.

\section{Author details}

${ }^{1}$ Division of Anesthesiology, Niigata University Graduate School of Medical and Dental Sciences, 1-757 Asahimachi-dori, Chuo Ward, Niigata, Niigata 951-8510, Japan. ${ }^{2}$ Department of Anesthesia, Nagaoka Chuo General Hospital, 2041 Kawasaki-cho, Nagaoka, Niigata 940-8653, Japan. ${ }^{3}$ Present address: Department of Anesthesiology, Uonuma Institute of Community Medicine, Niigata University Medical and Dental Hospital, 4132 Urasa, Minami-uonuma, Niigata 949-7302, Japan.

Received: 1 May 2015 Accepted: 14 July 2015

Published online: 27 August 2015

\section{References}

1. Cooper DW, Ryall DM, McHardy FE, Lindsay SL, Eldabe SS. Patient-controlled extradural analgesia with bupivacaine, fentanyl, or a mixture of both, after caesarean section. Br J Anaesth. 1996;76:611-5.

2. Fischer RL, Lubenow TR, Liceaga A, McCarthy RJ, Ivankovich AD. Comparison of continuous epidural infusion of fentanyl-bupivacaine and morphine-bupivacaine in management of postoperative pain. Anesth Analg. 1988:67:559-63.

3. Buggy DJ, Hall NA, Shah J, Brown J, Williams J. Motor block during patient-controlled epidural analgesia with ropivacaine or ropivacaine/ fentanyl after intrathecal bupivacaine for caesarean section. $\mathrm{Br} J$ Anaesth 2000;85:468-70.

4. Chen SY, Liu FL, Cherng YG, Fan SZ, Leighton BL, Chang HC, et al. Patient-controlled epidural levobupivacaine with or without fentanyl for post-cesarean section pain relief. Biomed Res Int. 2014;2014:965152.

5. Abboud TK, Dror A, Mosaad P, Zhu J, Mantilla M, Swart F, et al. Mini-dose intrathecal morphine for the relief of post-cesarean section pain—safety, efficacy, and ventilatory responses to carbon-dioxide. Anesth Analg. 1988;67:137-43.

6. Popping DM, Elia N, Marret E, Wenk M, Tramer MR. Opioids added to local anesthetics for single-shot intrathecal anesthesia in patients undergoing minor surgery: a meta-analysis of randomized trials. Pain. 2012;153:784-93.

7. Dahl JB, Jeppesen IS, Jorgensen H, Wetterslev J, Moiniche S. Intraoperative and postoperative analgesic efficacy and adverse effects of intrathecal opioids in patients undergoing cesarean section with spinal anesthesia: a qualitative and quantitative systematic review of randomized controlled trials. Anesthesiology. 1999;91:1919-27. 
8. Kato R, Shimamoto H, Terui K, Yokota K, Miyao H. Delayed respiratory depression associated with $0.15 \mathrm{mg}$ intrathecal morphine for cesarean section: a review of 1915 cases. J Anesth. 2008;22:112-6.

9. Hein A, Rosblad P, Gillis-Haegerstrand C, Schedvins K, Jakobsson J, Dahlgren G. Low dose intrathecal morphine effects on post-hysterectomy pain: a randomized placebo-controlled study. Acta Anaesthesiol Scand. 2012:56:102-9.

10. Gogarten W, Van de Velde M, Soetens F, Van Aken H, Brodner G, Gramke $H F$, et al. A multicentre trial comparing different concentrations of ropivacaine plus sufentanil with bupivacaine plus sufentanil for patient-controlled epidural analgesia in labour. Eur J Anaesthesiol. 2004;21:38-45.

11. Schewe JC, Komusin A, Zinserling J, Nadstawek J, Hoeft A, Hering R. Effects of spinal anaesthesia versus epidural anaesthesia for caesarean section on postoperative analgesic consumption and postoperative pain. Eur J Anaesthesiol. 2009;26:52-9.

12. Kim SH, Yoon KB, Yoon DM, Kim CM, Shin YS. Patient-controlled epidural analgesia with ropivacaine and fentanyl: experience with 2,276 surgical patients. Korean J Pain. 2013;26:39-45.

13. Amano E, Akamatsu T, Sonoda S, Konishi A, Shibuya H, Hirata T. Study of analgestic efficacy of ropivacaine-fentanyl patient-controlled epidural analgesia after upper abdominal gynecological surgery. Masui. 2006;55:1149-54.

14. Lockington PF, Fa'aea P. Subcutaneous naloxone for the prevention of intrathecal morphine induced pruritus in elective caesarean delivery. Anaesthesia. 2007:62:672-6.

15. Siddik-Sayyid SM, Yazbeck-Karam VG, Zahreddine BW, Adham AM, Dagher $\mathrm{CM}$, Saasouh WA, et al. Ondansetron is as effective as diphenhydramine for treatment of morphine-induced pruritus after cesarean delivery. Acta Anaesthesiol Scand. 2010;54:764-9.

16. Wu Z, Kong M, Wang N, Finlayson RJ, De Tran QH. Intravenous butorphanol administration reduces intrathecal morphine-induced pruritus after cesarean delivery: a randomized, double-blind, placebo-controlled study. J Anesth. 2012;26:752-7.

\section{Submit your manuscript to a SpringerOpen ${ }^{\circ}$ journal and benefit from:}

- Convenient online submission

- Rigorous peer review

- Immediate publication on acceptance

- Open access: articles freely available online

- High visibility within the field

- Retaining the copyright to your article

Submit your next manuscript at $>$ springeropen.com 